\title{
Standpunktfähigkeit und Perspektivenwechsel als Anforderung an die Lehrperson im konfessionell-kooperativen Lernsetting aus der Sicht von betroffenen Religionslehrkräften
}

\author{
Mirjam Zimmermann ${ }^{a}$ / Ulrich Riegel ${ }^{a}$ / Benedict Totsche ${ }^{a}$ / Steffi Fabricius ${ }^{a}$ \\ a Universität Siegen
}

Kontakt: zimmermann@evantheo.uni-siegen.de

eingereicht: 06.11.2020; angenommen: 04.12.2020

\begin{abstract}
Zusammenfassung: Der vorliegende Beitrag untersucht, wie Religionslehrpersonen aus NRW, die mit dem konfessionell-kooperativen Religionsunterricht vertraut sind, die Anforderung an sich selbst wahrnehmen, standpunktfähig zu sein und die Perspektive des Gegenübers einspielen zu können. Dazu wurden sieben Gruppeninterviews, 16 Briefe und 45 Fragebögen qualitativ-inhaltsanalytisch ausgewertet. Als Ergebnis wird festgehalten, dass die befragten Lehrkräfte Standpunktfähigkeit und Perspektivenwechsel eng aufeinander beziehen, wobei der Standpunktfähigkeit von den Befragten eine weit größere Aufmerksamkeit gewidmet wird. Standpunktfähigkeit wird entlang dreier Ebenen thematisiert, nämlich a) als religiöse Einstellung, b) als Wissen über die beiden Konfessionen und c) als Verhalten im Religionsunterricht. Für die Befragten gibt es nur auf der Wissens-Ebene die Möglichkeit, auch den Standpunkt der jeweils anderen Konfession einzunehmen. Darüber hinaus sehen sie im individuellen Glauben und in der kollektiven Perspektive der eigenen Kirche zwei mögliche Bezugspunkte für den eigenen Standpunkt. Allerdings wird auch gefragt, ob die Perspektive der Kirche nicht an sich vielgestaltig sein könne und inwiefern die Betonung eines Standpunkts zur „Re-Ideologisierung“ konfessionell-kooperativen Lernens führe. Schlagwörter: Konfessionell-kooperativer Religionsunterricht, Standpunktfähigkeit, Perspektivenwechsel, Religionslehrperson, Inhaltsanalyse
\end{abstract}

Abstract: This article examines how teachers of religion from North Rhine-Westphalia who are familiar with
cooperative religious education perceive the prerogative to be able to take a stand and to bring in the perspective
of their counterpart. For this purpose, seven group interviews, 16 letters and 45 questionnaires have been evaluated
qualitatively. As a result it is stated that in the perception of the participants the ability to take a stand and the
ability to take perspective are closely related to each other, whereby the ability to take a stand gets far more atten-
tion. The ability to take a standpoint is addressed on three levels, namely a) as religious attitude, b) as knowledge
about the denominations, and c) as behavior in the classroom. For the interviewees, only the knowledge level offers
the possibility of taking the perspective of the other denomination. Furthermore, they see two points of reference
for their own standpoint in their individual faith and in the collective perspective of their own church. However, it
is also asked whether the perspective of the church could not be in itself diverse and to what extent the emphasis
on one point of view leads to a "re-ideologization" of cooperative learning.
Keywords: Cooperative religious learning, taking a stand, taking perspective, teacher of religion, content analysis

Standpunktfähigkeit und Perspektivenwechsel sind zentrale Konzepte im konfessionell-kooperativen Religionsunterricht. Auf der einen Seite erfordert das Miteinander von zwei Konfessionen die Fähigkeit, sich in die Perspektive des Gegenübers hineinversetzen zu können. Auf der anderen Seite lebt gerade der konfessionelle Religionsunterricht von der Möglichkeit, die existentielle Tiefe religiöser Überzeugungen ins Unterrichtsgeschehen einbringen zu können. Um dieses Zueinander von Standpunktfähigkeit und Perspektivenwechsel im konfessionell-kooperativen Religionsunterricht (kokoRU) einzulösen, wird insbesondere von den Lehrpersonen gefordert, dass sie um ihren eigenen konfessionellen Standpunkt wissen und sich in die Perspektiven der Partnerkonfession hineinversetzen können. Der vorliegende Beitrag untersucht, wie Religionslehrpersonen, die in NRW kokoRU erteilen, diese Anforderungen beurteilen. Dazu werden die einschlägigen Aussagen aus sieben Gruppeninterviews, 16 Briefen und 45 
Fragebögen qualitativ-inhaltsanalytisch ausgewertet (3.). Zuvor wird die Bedeutung der Konzepte Standpunktfähigkeit und Perspektivenwechsel in der religionspädagogischen Diskussion um konfessionell-kooperatives Lernen herausgearbeitet (1.) und die Methode der Untersuchung beschrieben (2.). Den Beitrag beschließt eine Diskussion der Befunde (4.). ${ }^{1}$

\section{Zur Bedeutung von Standpunktfähigkeit und Perspektivenwechsel in der Religionspädagogik und im kokoRU}

Seit der evangelischen Denkschrift „Identität und Verständigung“ steht das Konzept konfessionell-kooperativen Lernens zur religionspädagogischen Diskussion. Programmatisch formuliert die Denkschrift: „In der Spannung von Identität und Verständigung ist die angemessene Gestalt des konfessionellen Religionsunterrichts für die $\mathrm{Zu}$ kunft die Form eines ,konfessionell-kooperativen Religionsunterrichts'. Weder legt er einfach zusammen, was nicht identisch ist, noch läßt er auseinanderfallen, was sich aufeinander verwiesen sehen sollte“ (EKD, 1994, S. 65). Seit diesem Impuls und Unterrichtsversuchen in Baden-Württemberg und Niedersachsen hat sich der konfessionellkooperative Religionsunterricht mittlerweile in diesen beiden Bundesländern und weiten Teilen Nordrhein-Westfalens als reguläre Organisationsform des konfessionellen Religionsunterrichts etabliert (Sajak, 2017).

Auch wenn eine detailliert ausformulierte Didaktik dieses Lernarrangements nach wie vor ein Desiderat darstellt (z. B. Platzbecker, 2018, S. 53; Schröder \& Biesinger, 2016, S. 85), beruht der kokoRU prinzipiell auf der Verschränkung konfessioneller Perspektiven mit dem Zweck, dass die Schülerinnen und Schüler die Sichtweisen der jeweils anderen Konfession besser verstehen und sich gleichzeitig ihres eigenen konfessionellen Standpunkts bewusst werden und diesen reflektieren (Lindner \& Simojoki, 2018, S. 93; Woppowa, 2015; 2017). Es soll erreicht werden, dass die Kinder und Jugendlichen die Konfessionen ,,in angemessener Form beschreiben können, sie miteinander in Beziehung bringen und sich selbst dazu ins Verhältnis setzen können“ (Schweitzer, Biesinger, Conrad \& Gronover, 2006, S. 97). Dazu soll die konfessionelle Begegnung im Unterricht „authentisch“ sein, was dann erreicht wird, „wenn eine Konfession von den ihr angehörigen Interaktionspartnern so dargestellt wird, dass die Anderskonfessionellen sie verhältnismäßig vollständig und unverzerrt wahrnehmen können und wenn die Darstellungsweise außerdem so geartet ist, dass keine direkt oder indirekt abqualifizierenden Impulse bezüglich der anderen Konfession mitkommuniziert werden“ (Kuld, Schweitzer, Tzscheetzsch \& Weinhardt, 2009, S. 60; auch DBK, 2016, S. 32). Neben derartigen persönlichen Erfahrungen spielen im kokoRU aber auch konfessionelle Perspektiven, die durch das Unterrichtsmaterial in die Auseinandersetzung eingespielt werden, eine zentrale Rolle. Zeugen persönliche Erfahrungen in der Regel von der individuellen Rezeption konfessioneller Positionen, eröffnet das Unterrichtsmaterial darüber hinaus auch die Möglichkeit, konfessionelle Positionen im Sinn der theologischen und kulturellen Traditionen der Religionsgemeinschaften zu thematisieren (Bauer, 2019, S. 432). Gleichzeitig hilft das Unterrichtsmaterial den Schülerinnen und Schülern, einen Zugang zu beiden Konfessionen zu finden, die kaum bis gar nicht auf konfessionsspezifische Erfahrungen zurückgreifen können oder aber auch (noch) nicht in der Lage sind, solche Erfahrungen angemessen auszudrücken. „Konfessionelle Stile und Prägungen in einem ökumenischen Horizont identifizieren, deuten, beurteilen und gestalten zu können, braucht authentische Sprechsituationen [...], ein Wissen um Vorurteile und um die Versuchung von Fremdzuschreibungen [...], den besonderen Respekt vor der Minderheitskonfession [...], das Ineinander von Positionalität und Austausch [...], Perspektivenwechsel und Korrelation“ (Schambeck \& Schröder, 2017, S. 351-359). In der Summe ist guter kokoRU demnach keine Konfessionskunde, sondern legt konfessionelle Prägungen und Positionen offen und thematisiert diese explizit, wobei dafür auf konfessionelle Erfahrungen der einzelnen Person zurückgegriffen wird (Schweitzer et al., 2006, S. 100f.).

In diesem didaktischen Arrangement sind die Religionslehrpersonen in besonderer Weise gefordert. Sie haben konfessionelle Differenz nicht nur als bildsam zu inszenieren, indem sie z. B. auf passendes Material zurückgreifen, sondern stehen durch ihren kirchlichen Auftrag für eine institutionell gebundene konfessionelle Perspektive in diesem Arrangement ein. „Die Religionslehrkräfte unterrichten konfessionsbewusst und differenzsensibel und sind als katholische oder evangelische Lehrkräfte erkennbar“ (DBK, 2016, S. 33). Die Lehrpersonen sollen nicht nur eine einschlägige inhaltliche Expertise in den beiden zu unterrichtenden christlichen Traditionen mitbringen, sondern auch die Teilnehmerperspektive, die die Bekenntnisdimension des Christlichen exemplarisch zum Ausdruck bringt, in den Unterricht einspielen. „Lehrerinnen und Lehrer sind deshalb weit mehr als Begleiter oder Moderatoren. Sie repräsentieren den spezifischen Weltzugang ihres Faches. Sie bringen ein, was im Text nicht steht, was aber

\footnotetext{
${ }^{1}$ Wir bedanken uns ausdrücklich für die kritisch-konstruktiven Hinweise der beiden Gutachten, die wir gerne im vorliegenden Beitrag übernommen haben.
} 
für sein Verständnis von zentraler Bedeutung ist“ (Schmid, 2017, S. 64). Die Lehrkräfte im kokoRU sollten sich somit ihres eigenen konfessionellen Standpunkts bewusst sein, weil sie im kooperativen Lernarrangement nochmals stärker in ihrer konfessionellen Rückbindung herausgefordert sind. Friedrich Schweitzer (2017) erinnert in diesem Zusammenhang, dass in evangelischer Perspektive jegliches Bekenntnis an die eine Kirche Jesu Christi rückgebunden ist und jegliche Konfessionalität damit in ökumenischem Geist über sich selbst hinausweist. Die konfessionelle Verortung der Lehrperson zeugt deshalb vor allem von deren sozialer und kultureller Verortung im religiösen Bereich, nicht jedoch von einer Exklusivität ihres Bekenntnisses. Im katholischen Verständnis beinhaltet das Konzept der "gesprächsfähigen Identität“ (DBK, 1996, S. 49) zwar ebenfalls eine Offenheit anderen Lesarten des christlichen Vermächtnisses gegenüber, fokussiert aber nochmals stärker das Spezifische der römisch-katholischen Lesart dieses Vermächtnisses und der darin im kirchlichen Sinn eingeschriebenen „Hierarchie der Wahrheiten“ (DBK, 2016, S. 32). Jan Woppowa warnt in dieser Hinsicht vor einer „konfessionellen Überbeanspruchung“ (2021, S. 203), die die Individualität der konfessionellen Verortung der Lehrkraft übersieht und ihr eine zu starke Repräsentation des Institutionellen aufbürdet.

Auch aus empirischer Perspektive scheint dieser Anspruch für die Lehrpersonen in mehrfacher Hinsicht eine Heraus- bis Überforderung darzustellen. So berichten Hans-Günter Heimbrock und Felix Kerntke, dass „professionelles Handeln und dessen theologische Begründung [...] tendenziell auseinander" fallen (2017, S. 36). Den befragten Lehrpersonen gelinge es zwar, ihren Unterricht konfessionell zu gestalten, immer weniger jedoch, diese Konfessionalität inhaltlich zu begründen (vgl. ähnliche Tendenzen im Projekt der Essener Forschungsgruppe, die aus einem anderen Erkenntnisinteresse heraus vielen untersuchten Religionsstunden eine fehlende Positionalität attestiert; Englert, Hennecke \& Kämmerling, 2014). Analog dazu verstehen sich 75\% der befragten evangelischen Religionslehrpersonen aus Schleswig-Holstein nicht als Vertreter*in der evangelischen Kirche, 65\% erachten sich nicht als authentisches Beispiel ihrer Konfession und 58\% geben an, im Unterricht nicht den evangelischen Glauben zu vertreten (Pohl-Patalong, Woyke, Boll, Dittrich \& Lüdke, 2016, S. 337). In der Befragung von Religionslehrpersonen aus Nordrhein-Westfalen dominieren lebensweltliche und dialogische Zielvorstellungen für den Religionsunterricht, wogegen Ziele, die den konfessionellen Charakter des Religionsunterrichts betreffen, auf den hinteren Plätzen der Beliebtheitsskala rangieren (Rothgangel, Lück \& Klutz, 2017, S. 38-47), auch wenn die Bedeutung „,konfessioneller Bindung und Prägung“ (ebd., S. 121) für die Befragten groß ist. Christiane Caspary findet in Daten zum konfessionell-kooperativen Unterricht in Baden-Württemberg, dass die „Lehrpersonen bei der Darstellung konfessioneller Differenz offensichtlich zu einem Modus der Mitteilung von Religion im Unterricht über[gehen]“ (Caspary, 2016, S. 191), sodass „konfessionelle Differenz dadurch vermutlich eher den Status einer Behauptung [hat], einer religiösen Anschauung und weniger einer Praxis religiöser Weltdeutung oder religiöser Vollzüge“ (ebd., S. 187). Allerdings berichten einige Lehrpersonen auch, dass ihre Schülerinnen und Schüler durch das kooperative Setting nun wenigstens wüssten, welcher Konfession sie angehören (Isak, 2009, S. 138). Wenn es um den eigenen Standpunkt geht, zeigen die katholischen Kinder einer Evaluation aus Baden-Württemberg eine eher stärkere konfessionelle Identität, während sich die evangelischen Befragten ihres Eigenen weniger bewusst zu sein scheinen (Schweitzer \& Biesinger, 2002, S. 170-174). Allerdings erweist sich das Wissen vieler Befragter in dieser Evaluation sowohl über die andere als auch über die eigene Konfession als stark klischeehaft ausgeprägt (ebd., S. 174-175). In dieser Hinsicht ist es bemerkenswert, dass die wenigen vorliegenden empirischen Indizien andeuten, dass die praktische Arbeit mit den Kolleginnen und Kollegen der anderen Konfession im konfessionell-kooperativen Religionsunterricht eher kaum zur Klärung der eigenen Position oder zur Aufklärung über den Glauben der anderen Konfession führt (z. B. Isak, 2009; Gennerich \& Mokrosch, 2016, S. 71).

Fasst man den bisherigen Forschungsstand zusammen, erweist sich die Verschränkung von Perspektivenwechsel und Standpunktfähigkeit als ein spezifisches Charakteristikum des kokoRU. Diese Verschränkung betrifft nicht nur die didaktische Inszenierung von Unterricht, sondern aus ihr erwachsen auch spezifische Anforderungen an die Lehrperson. Zu Letzterem ergeben erste empirische Einsichten ein ambivalentes Bild. Auf der einen Seite wird das konfessionell-kooperative Wechselspiel zwischen Eigenem und Anderem mehrheitlich als positiv eingeschätzt, auf der anderen Seite erscheint im Handlungsrepertoire der Lehrpersonen das Zueinander von Perspektivenwechsel und Positionalität nur ansatzweise ausgeprägt. Angesichts der Tatsache, dass die Daten der hier berichteten Erfahrungen oft deutlich älter als das Publikationsdatum und somit mindestens zehn Jahre alt sind und die Diskussion um den kokoRU in den letzten zehn Jahren auch die Öffentlichkeit vieler Fachkollegien erreicht hat, stellt sich die Frage, wie sich das Zusammenspiel von Perspektivenwechsel und Standpunktfähigkeit im professionellen Verständnis von Religionslehrpersonen heute darstellt. Im Folgenden wird deshalb untersucht, wie Lehrpersonen des kokoRU in NRW dieses Zusammenspiel erfahren und evaluieren. 


\section{Zur Anlage der Studie: Datengrundlage und Methodik}

Die im Folgenden verwendeten Daten entstammen einem größeren Projekt zur Auswertung des konfessionell-kooperativen Religionsunterrichts (kokoRU) in NRW, wobei für diesen Beitrag ausschließlich die qualitativen Daten ausgewertet werden. Sie speisen sich aus drei Quellen, nämlich Gruppeninterviews im Rahmen einer sog. „Regionalkonferenz", Briefen an das Schulministerium und die beteiligten Kirchenleitungen am Ende dieser Konferenz sowie einer schriftlichen Befragung von Moderatorinnen und Moderatoren, welche die Einführung dieser Organisationsform des Religionsunterrichts in NRW unterstützen.

Besagte Regionalkonferenz fand am 3. März 2020 in Düsseldorf mit dem Ziel statt, Erfahrungen von Religionslehrpersonen mit der Praxis des kokoRU auszutauschen. Nach einer Einführungsrunde wurden zuerst Fragen zur Organisation dieser Unterrichtsform in Kleingruppen diskutiert, dann in einer zweiten Runde der Umgang mit der Positionalität dieses Ansatzes. Zum Abschluss der Konferenz hatten die Teilnehmenden Gelegenheit, anonym einen Brief zu verfassen, der dem Schulministerium und den beteiligten Kirchenleitungen ein Zwischenfeedback zum kokoRU mitteilt.

Zur Regionalkonferenz wurde offen eingeladen. Die nach Anmeldeschluss noch nicht belegten Plätze wurden gemäß dem theoretical sampling durch gezielt angesprochene Personen ergänzt. Angestrebt war eine gleichmäßige Verteilung der Teilnehmenden nach Geschlecht, Konfession und Schulstufe. Tatsächlich ergab sich in allen Kategorien eine Verteilung von 13:9, d. h. es haben 13 Grundschul- und 9 Sekundarschullehrkräfte, 13 Frauen und 9 Männer, sowie 13 katholische und 9 evangelische Lehrpersonen teilgenommen. Alle Teilnehmenden haben Erfahrungen mit dem kokoRU, zum größten Teil aus eigener Unterrichtspraxis, in einigen Fällen durch ihre Moderatorentätigkeit. Außerdem kann angenommen werden, dass das Interesse der Teilnehmenden an dieser Organisationsform religiösen Lernens überdurchschnittlich ausgeprägt ist, denn sie haben sich eigens für diese Regionalkonferenz von ihren Schulen beurlauben lassen.

Bei der Einteilung der Kleingruppen (vormittags: 2x Primarstufe und 2x Sekundarstufe; nachmittags: 2x Primarstufe und 1x Sekundarstufe) wurde auf ein einigermaßen ausgeglichenes Konfessionsverhältnis geachtet. Die Kleingruppen hatten jeweils ca. eine Stunde zur Diskussion anhand von Leitfragen. Außer den Lehrerinnen und Lehrern selbst waren keine weiteren Personen im Raum. Die Diskussionen wurden aufgenommen und nach elementaren Regeln transkribiert.

Ferner nutzten viele Lehrpersonen die Gelegenheit, am Ende der Regionalkonferenz einen Brief an das Schulministerium und die beteiligten Kirchen zu verfassen, in dem sie Stärken und Schwächen des kokoRU aufzeigen. Hier liegen 16 Briefe vor, wobei diese vollständig anonym erstellt wurden und das Teil-Sample dieser Datenquelle nicht näher bestimmt werden kann.

Die dritte Datenquelle bilden schriftliche Befragungen der Moderatorinnen und Moderatoren, die bereits zu Projektbeginn zwischen Mai und Juni 2019 durchgeführt wurden. Hierzu wurden alle einschlägigen Personen in NRW angeschrieben mit der Bitte, anhand von offenen Leitfragen die Chancen und Grenzen des kokoRU einzuschätzen. 45 ausgefüllte Fragebögen liegen vor (Rücklaufquote: 54\%), wovon 23 von Männern und 22 von Frauen stammen. 27 der Befragten sind katholisch und 18 evangelisch.

In der Summe kann die vorliegende Analyse auf sieben Gruppeninterviews, 16 Briefe und die Antworten von 45 Fragebögen zurückgreifen. Alle Texte wurden in MAXQDA eingespeist und qualitativ-inhaltsanalytisch gemäß dem zusammenfassenden Typus ausgewertet (Mayring, 2015). Dazu wurde zuerst eine thematische Sinneinheit als Analyseeinheit festgelegt, was bei den Fragebögen sehr oft die komplette Antwort auf eine Leitfrage ist, bei den Gruppeninterviews unter Umständen eine kürzere Sequenz mit Rede und Gegenrede. Jeder Analyseeinheit wurden anschließend Codes zugewiesen, die sich auf zentrale Textstellen in den Analyseeinheiten bezogen. Dabei waren Mehrfachkodierungen innerhalb einer Einheit erlaubt, um der Komplexität des Materials gerecht zu werden. Nachdem das komplette Material kodiert war, wurden inhaltsanaloge Codes zu Kategorien zusammengefasst. Für diesen Beitrag wurde der Code „Lehrerinnen und Lehrer“ (191 Einheiten) herangezogen, außerdem die Codes „Positionalität“ oder „Konfessionalität“ (89 Einheiten). Diese Belegstellen wurden anschließend nach den Aspekten „Standpunktfähigkeit“ und „Perspektivenwechsel“ kategorisiert. Nach dieser Auswahl blieben 30 Analyseeinheiten übrig. Sie wurden dann gemäß der in ihnen angesprochenen Themen Sub-Kategorien zugeteilt. Anschließend wurde die dimensionale Ausprägung innerhalb der Sub-Kategorien bestimmt. Auf diese Weise entstand ein differenziertes Bild der Äußerungen der befragten Lehrpersonen zum Zusammenspiel von Perspektivenwechsel und Standpunktfähigkeit im kokoRU in NRW. 


\section{Analyse der qualitativen Daten}

Im Folgenden stellen wir die Analyse der qualitativen Daten vor. Dabei kann als erste Einsicht herausgestellt werden, dass die wenigen Aussagen, die mit Perspektivenwechsel kodiert wurden, in einem engen Bezug zu Indikatoren stehen, die eine Standpunktfähigkeit andeuten. Deshalb wird der Perspektivenwechsel in der folgenden Analyse als eine Sub-Kategorie der Standpunktfähigkeit behandelt. In der Folge lassen sich unter den Belegen vier SubKategorien unterscheiden, nämlich

- Bedeutungen von Standpunktfähigkeit (3.1),

- Bezugspunkte von Standpunktfähigkeit (3.2) und

- Akzeptanz und Ablehnung von Standpunktfähigkeit (3.3),

- Perspektivenwechsel im Kontext von Standpunktfähigkeit (3.4).

Diese vier Sub-Kategorien werden im Folgenden näher beschrieben.

\section{I Sub-Kategorie: Bedeutungen von Standpunktfähigkeit}

Die erste Analysekategorie ergründet die Art und Weise, welche Bedeutung die befragten Lehrpersonen dem Konzept „Standpunktfähigkeit“ zuschreiben bzw. welche Funktion sie ihm im konfessionell-kooperativen Lernsetting zusprechen. In dieser Hinsicht lassen sich im Wesentlichen drei Grundkonstellationen antreffen, denn Standpunktfähigkeit wird entweder

- als eine Einstellung bzw. Haltung der Lehrperson betrachtet, oder

- als eine Aktionsform der Lehrperson im Unterrichtsprozess, oder

- als eine Wissens-Kategorie.

In den ersten beiden Grundkonstellationen finden sich wiederum jeweils zwei unterschiedliche Ausprägungen. So kann man bei der Auffassung von Standpunktfähigkeit als Einstellung bzw. Haltung nochmals differenzieren, ob die Standpunktfähigkeit als Voraussetzung bzw. Grundlage oder als Effekt der konfessionell-kooperativen Organisationsform angesehen wird.

Viele Aussagen betrachten eine konfessionelle Identität als Voraussetzung der Lehrperson innerhalb eines konfessionell-kooperativen Lernarrangements. In diesen Äußerungen trifft man auf eine Logik gemäß der man erst um die konfessionellen Gemeinsamkeiten und Differenzen wissen und sich seiner eigenen Position innerhalb dieses Spektrums vergewissern muss, um überhaupt kompetent einen kokoRU erteilen zu können. Einschlägige Beispiele dieser Ausprägung sind:

"Also nur so kann ich ja Religionsunterricht erteilen, wenn ich authentisch bin."

"Sich der eigenen Konfessionalität als Lehrkraft bewusst zu werden, ist für mich die wichtigste Grundlage."

In manchen Aussagen, in denen Standpunktfähigkeit als Einstellung oder Haltung verwendet wird, ergibt sich diese als Konsequenz des konfessionell-kooperativen Lernarrangements. In der Regel wird argumentiert, dass die Vorbereitung auf diese Organisationsform eine Auseinandersetzung mit konfessionellen Positionen zur Folge hat und sich aus dieser Auseinandersetzung heraus auch ein konfessionelles Bewusstsein formt. Standpunktfähigkeit wäre dann ein Effekt dieser Organisationsform des Religionsunterrichts. In den Belegen finden sich hierzu u. a. die folgenden Beispiele:

„Dass man [in der Arbeit an den Curricula für den kokoRU] so ein eigenes Standing auch dafür bekommt: Wer bin

ich denn? Auch mit meiner Religiosität?"

"Ich meine, was ja wohl die Modellversuche gezeigt haben, was die Lehrkräfte in der Evaluation berichtet haben auch,

schon sehr früh, dass im Grunde genommen das konfessionelle Bewusstsein vor allem bei den Lehrkräften dadurch nochmal angeschärft wurde."

Weiterhin wird die Standpunktfähigkeit auch als Aktionsform im Unterrichtsgeschehen angesprochen. Es geht in diesen Fällen um die Präsenz einer konfessionellen Position in der unterrichtlichen Auseinandersetzung. Auch hier lassen sich zwei Ausprägungen unterscheiden. Auf der einen Seite sprechen die Befragten davon, dass sie selbst eine derartige kirchliche Position ins Unterrichtsgeschehen einspielen. Standpunktfähigkeit fungiert somit als Ressource, aktiv im Religionsunterricht zu agieren. Ein paradigmatisches Beispiel dieser Ausprägung ist:

"[...] das hat ja was von persönlicher Zeugenschaft, ich gebe Auskunft über meinen Glauben, ich gebe darüber Aus-

kunft, wie es in meiner Kirche, egal ob katholisch oder evangelisch, gedacht ist."

Die Befragten berichten aber auch von Situationen, in denen sie mit ihrem Glauben identifiziert wurden bzw. ihnen ihre eigene Konfessionalität von Dritten zugeschrieben wird. Standpunktfähigkeit ist in dieser Verwendung eine Reaktion auf soziale Zuschreibung. O-Töne dieser Ausprägung sind: 
"Das einzige Mal, wo ich wirklich mal mit meiner katholischen Authentizität auch vor den Schülern gestanden habe, das ist immer der Augenblick, wo ich bete, weil ich mache natürlich das Kreuzzeichen."

„Die wollen von mir wissen: ,Welche Positionen vertrittst du, vertreten Sie persönlich?' Die fragen mich ganz konkret: ,Gehen Sie eigentlich sonntags in die Kirche?' Und dann muss ich Butter bei die Fische tun. ,Ja. Ja, doch.' Da darf ich auch nicht lügen. Und wenn ich dann sage/ wenn ich dann stehe als Vertreter der katholischen Kirche und sage: ,Nein, eigentlich nicht.' Dann habe ich verloren. Dann ist vorbei. Dann kann ich erzählen, was ich will. ,Was erzählen Sie mir denn da? Sie glauben ja selber nicht dran."

Schließlich wird Standpunktfähigkeit in wenigen Fällen mit konfessionsspezifischem Wissen identifiziert. Diese Belege verdanken sich der Erfahrung, dass von einer Lehrperson erwartet wird, sich zumindest in der eigenen Konfession gut auszukennen und kundig über die Sachverhalte derselben Auskunft geben zu können. Einschlägige Beispiele sind:

"Und kenn' ich mich mit allem auch sehr gut aus? Und kann ich auch die Fragen, die Kollegen dann ja auch haben, alle beantworten?"

"Mir geht es nicht darum, das Trennende hervorzuheben. Aber ich muss wissen, wovon ich spreche ( $z$. B. Kirchenverständnis, Ämter, Strukturen der eigenen Kirche). Nur mit dieser Klarheit kann eine Lehrkraft verantwortlich RU erteilen."

\subsection{Sub-Kategorie: Bezugspunkte von Standpunktfähigkeit}

Ferner finden sich in den Aussagen der Befragten zwei unterschiedliche Bezugspunkte für Standpunktfähigkeit, denn diese kann sich sowohl auf die persönliche Religiosität beziehen als auch auf institutionelle Positionen. Beide Bezugspunkte werden z. B. im oben bereits zitierten O-Ton sichtbar, in dem es um den sonntäglichen Kirchgang geht. Weiterführend erscheint uns, dass einige Befragte beide Referenzpunkte zueinander in Beziehung setzen. Dabei lassen sich zwei Konstellationen unterscheiden, denn individuelle Religiosität und institutionelle Positionen können miteinander harmonieren oder im Konflikt miteinander stehen. Ein harmonisches Verhältnis wird in den folgenden Aussagen deutlich, die beide Referenzpunkte entweder kommentarlos nebeneinanderstellen oder explizit als miteinander identisch ausweisen:

"[...] das hat ja was von persönlicher Zeugenschaft, ich gebe Auskunft über meinen Glauben, ich gebe darüber Aus-

kunft, wie es in meiner Kirche, egal ob katholisch oder evangelisch, gedacht ist."

"Und wenn ich dann sagel wenn ich dann stehe als Vertreter der katholischen Kirche und sage: ,Nein, eigentlich nicht.'

Dann habe ich verloren. Dann ist vorbei."

Es finden sich aber auch Aussagen, in denen individuelle Religiosität und institutionelle Positionen explizit in Konflikt miteinander stehen bzw. nicht notwendig miteinander harmonieren müssen, wie folgende Aussagen andeuten:

"Ja, ich meine, was ist die Wahrheit, die ich als Religionslehrer für meinen Glauben bezeuge, so, ne? Und wenn ich als katholischer Religionslehrer bezeuge, ich habe hohe Sympathiewerte an gewissen Stellen für die katholische Kirche, kann aber mit der Sexualmoral nichts anfangen, dann ist das mein Zeugnis. Punkt. So. Das ist die Wahrheit, für die ich stehe."

"Ich habe einige katholische Kollegen, die ein Problem mit dem Papst haben, aber es trotzdem vermitteln müssen. Die kommen natürlich in eine Situation oder können in eine Situation kommen, wo es denen vielleicht nicht so gut geht."

Inwiefern die Beobachtung, dass es sich in den beiden letzten Belegstellen um Beispiele römisch-katholischer Konfessionalität handelt, den Schluss erlaubt, dass es sich bei den Konfliktfällen um eine konfessionsspezifische Konstellation handelt, kann aufgrund des Datenmaterials nicht entschieden werden. Auf der einen Seite scheinen es in unserem Datenmaterial ausschließlich römisch-katholische Kolleg*innen zu sein, die solche Äußerungen vorbringen oder über die solche Äußerungen getroffen werden. Allerdings ist dieses Material nicht repräsentativ.

\subsection{Sub-Kategorie: Akzeptanz und Ablehnung von Standpunktfähigkeit}

Unabhängig von der konkreten Verwendung des Konzepts der Standpunktfähigkeit und seiner Bezugspunkte, finden sich im Textmaterial positive und negative Bezüge darauf. Demnach lassen sich entlang der Akzeptanz-Dimension drei Ausprägungen identifizieren. Die erste Ausprägung bezieht sich positiv auf das Konzept der Standpunktfähigkeit im konfessionell-kooperativen Lernarrangement. In der Regel wird sie entweder als Voraussetzung für oder als ein Effekt von kokoRU angesprochen (vgl. oben). Typische Aussagen dieser Ausprägung sind:

"Sich der eigenen Konfessionalität als Lehrkraft bewusst zu werden, ist für mich die wichtigste Grundlage für einen angemessenen KoKoRU."

"Gut ist, dass sich die Lehrkräfte mit der eigenen Konfession auseinandersetzen [...]." 


\section{"[...] da sie im KoKoRu eine reflektierte Haltung zu eigenen konfessionellen und religiösen Prägungen einzunehmen gezwungen sind [...]."}

Es finden sich in den Belegstellen aber auch zwei Aussagen, die eine solche Standpunktfähigkeit in Frage stellen. Die erste Anfrage speist sich aus der Vermutung, dass eine Lehrperson, die im Unterricht einen konfessionellen Standpunkt bezieht, - zumindest "fast“ - zu einer „Ideologisierung“ des Religionsunterrichts führen würde. Standpunktfähigkeit wird hier somit als Interaktionsstrategie kritisiert, weil sie ein Unterrichtsziel fördern würde, das von der betroffenen Lehrperson nicht geteilt wird. Dieser Bezug findet sich in einer Passage einer Gruppendiskussion:

„Wenn ich aber anfange, plötzlich zu meinen, ich trete jetzt plötzlich mal wieder als evangelischer oder katholischer Religionslehrer oder Religionslehrerin auf, dann habe ich, glaube ich, etwas falsch verstanden, was jetzt konfessionelle Kooperation ausmacht. [...] Also wenn das wirklich so ist, das wäre ja fast schon eine Ideologisierung von Religionsunterricht."

Eine zweite Anfrage an das Konzept der Standpunktfähigkeit erwächst aus der Beobachtung, dass die Konfessionen vielgestaltig sind und es deshalb eine monolithische konfessionelle Identität, auf die sich ein solcher Standpunkt bezieht, nicht geben könne. Standpunktfähigkeit kann damit in letzter Konsequenz zu einer Homogenisierung innerkonfessioneller Vielfalt führen. Die einschlägige Passage aus einer Gruppendiskussion lautet folgendermaßen:

„Ob man letztlich diese Frage danach, wie man sich positioniert als Lehrkraft mit seiner ganzen Perönlichkeit, mit seinem eigenen Glauben, als Zeuge des Glaubens, wie es ja bei uns heißt, ob das nicht sowieso am Ende schon eine relativ große Heterogenität ergibt, die man am Ende wahrscheinlich gar nicht mit der Konfession als Merkmal abgrenzen kann. Also wenn ich an die Kolleginnen und Kollegen denke, die ich im katholischen Unterricht erlebe, und das, wenn ich an meine Schule gucke, auch evangelischerseits, dann entdecke ich da eine so große Bandbreite an unterschiedlichen Einstellungen und Haltungen, auch an unterschiedlichen Theologien, die da praktiziert und auch gelehrt wird, dass ich mich frage, ob sich nicht da sowieso schon eine so große Bandbreite auftut, dass diese Grenze oder dieses Merkmal "Konfession' im Grunde verschiedenen Religionsunterrichten so ausmacht."

In den beiden zuletzt genannten Ausprägungen erweist sich Standpunktfähigkeit somit als eine problematische Anforderung innerhalb des konfessionell-kooperativen Arrangements.

\subsection{Sub-Kategorie: Perspektivenwechsel im Kontext von Standpunktfähigkeit}

Wie einleitend bemerkt, steht der Perspektivenwechsel für die Befragten in einem engen Bezug zur Standpunktfähigkeit. Faktisch geht es in allen einschlägigen Äußerungen um die Möglichkeit, angesichts der eigenen konfessionellen Verortung angemessen die Positionen der anderen Konfession übernehmen zu können. Paradigmatisch für dieses Problembewusstsein steht die folgende Aussage:

„Es war lange nicht klar, wie genau die Rolle der jeweils konfessionsgebundenen Lehrkraft in einer konfessionsheterogenen Lerngruppe aussehen soll. Die eigene, individuelle konfessionelle Sichtweise spielte dabei keine entscheidende Rolle; vielmehr rückte die Frage: ,Kann ich überhaupt über die andere Konfession informieren bzw. interkonfessionelle Lernarrangements bereitstellen, ohne dabei konfessionelle Feinheiten potentiell misszuverstehen?' in den Vordergrund."

Als mögliche Antwort auf diese Frage finden sich im Textmaterial drei unterschiedliche Typen. Der erste Typ verneint die Möglichkeit, die andere Konfession angemessen darzustellen, grundsätzlich. Demnach ist die eigene konfessionelle Verortung so stark bzw. die Sache des Religionsunterrichts so tiefgreifend konfessionell geprägt, dass es keine Möglichkeit gibt, als Außenstehende im Religionsunterricht angemessen über das andere zu sprechen. Exemplarisch für eine solche Haltung steht der folgende, im O-Ton mit viel Emotion vorgetragene Gesprächsausschnitt:

"G1: Also, ich mache evangelischen Religionsunterricht, fertig, aus. Mit meiner Position.

G2: Ich mache keinen katholischen.

G3: Ich kann nicht katholisch unterrichten.

G2: Genau. Und du machst katholischen Religionsunterricht.

G4: Genau. Mit meiner Position.

G2: Genau."

In einer Spielart dieses Typs wird der Lehrerwechsel als operativer Modus vorgeschlagen, mit diesem Dilemma im Unterricht konstruktiv umzugehen. Demnach informiert die katholische Lehrkraft über den katholischen Zugang zum Sachverhalt und die evangelische über den evangelischen, wobei beide in der Lage sind, die ihren eigenen Perspektiven innewohnende Positionalität zum Ausdruck zu bringen. In einer anderen Gruppendiskussion wird diese Option folgendermaßen beurteilt: 
„Und beide geben auf ihre Weise Zeugnis und stehen als Zeugen den Schülern sozusagen Rede und Antwort."

Der zweite Typ verneint ebenfalls einen Perspektivenwechsel, führt dafür aber keine prinzipiellen Gründe an, sondern argumentiert mit seiner fehlenden Bedeutung in der unterrichtlichen Praxis. Demnach gibt es konfessionelle Unterschiede, die man grundsätzlich auch unabhängig von der eigenen Konfession rekonstruieren kann, die für den konkreten Unterricht aber zu feinsinnig oder anderweitig ohne Bedeutung sind, sodass ein Perspektivenwechsel in der Unterrichtsvorbereitung oder im Unterricht selbst keine Rolle spielen. Eine einschlägige Aussage liest sich folgendermaßen:

"So, und jetzt sage ich mal schlichtweg: Martin, ja? Das ist in $x x$ das Thema an und für sich, fertig aus, egal, ob ich jetzt evangelisch bin oder katholisch. Und jetzt ganz ehrlich, ich glaube nicht, dass die katholische Seite jetzt ein völlig anderes Bild vom Martin hat, als es die anderen tun, als es die evangelische Seite tut. So unterschiedlich nicht. Es ist da ein Unterschied, ja. Aber er spielt doch im Unterricht weniger Rolle. [...] Ich mache mir doch keine Gedanken über eine andere Sichtweise."

Im dritten Antworttyp wird bei dem Perspektivenwechsel unterschieden zwischen einer Ebene der Überzeugungen und einer Ebene der Sachverhalte. Demnach muss dieser Wechsel auf der Überzeugungsebene scheitern, weil diese existentiell verwurzelt ist und man Überzeugungen nicht beliebig tauschen kann. Auf der Ebene der Sachverhalte ist ein Perspektivenwechsel dagegen möglich, denn man kann lernen, wie sich ein Sachverhalt bei der anderen Konfession darstellt bzw. wie die andere Konfession einen Sachverhalt einschätzt. Im Religionsunterricht kann dieser Wechsel im Modus der Information zur Kenntnis gegeben werden. Eine Kollegin wählt für diesen Modus den Begriff der „Religionskunde“. Ihre Aussage, die exemplarisch für diesen Typ ist, lautet:

"Also nur so kann ich ja Religionsunterricht erteilen, wenn ich authentisch bin. So. Und dann ist völlig klar: Du bist katholisch und ich bin eben evangelisch. Und natürlich habe ich immer mal im Blick, und ich werde auch immer wieder gefragt, das ist der Grundschule vielleicht anders: ,Was sagt der Papst dazu?' Oder: ,Haben Sie gelesen? Die Bischöfel' Oder so, ne? Dann kann ich immer nur sagen, ich kann eigentlich nur das so sagen, was ich mir selber ein Stück weit angeeignet habe von der anderen Konfession. Aber mehr geht da auch nicht. Und das ist für mich eine Form von Religionskunde, die ich dann den Schülern weitergebe. Aber ich gehe nicht hin und sage: ,Ich bin evangelisch und jetzt muss ich aber katholisch im Hinterkopf haben, weil es ist jetzt KoKoRU.'"

\section{Diskussion}

In diesem Beitrag wurde anhand der qualitativen Daten im Evaluationsprojekt zum kokoRU in NRW untersucht, wie die Lehrpersonen dieser Organisationsform von Religionsunterricht die Anforderung an sich selbst wahrnehmen, standpunktfähig zu sein und die Perspektive des Gegenübers einspielen zu können. Wegen des qualitativen Charakters der Analyse können nur Aussagen zur Ausprägung dieser Wahrnehmung gemacht werden. Wie verbreitet eine jeweilige Wahrnehmung ist oder auf welchen Einflussfaktoren sie beruhen könnte, lässt der gewählte inhaltsanalytische Blick nicht zu. Unter dieser Prämisse sind die eben beschriebenen Befunde zu diskutieren.

Eine erste zentrale Erkenntnis ist, dass die befragten Lehrkräfte Standpunktfähigkeit und Perspektivenwechsel eng aufeinander beziehen. In vielen Aussagen zur Standpunktfähigkeit spielen die Präsenz der anderen Konfession und die Notwendigkeit, dieser gerecht zu werden, eine Rolle. Umgekehrt findet die Fähigkeit, die Perspektive der anderen Konfession zu übernehmen, in vielen Aussagen ihre Grenze im existentiellen Charakter von Konfessionalität, aus der sich auch die Standpunktfähigkeit speist. Standpunktfähigkeit und Perspektivenwechsel erscheinen damit in der Wahrnehmung der Befragten als enge Korrelate, was deren Verwendung in der religionspädagogischen Diskussion entspricht. Auch hier sind beide Konzepte eng aufeinander bezogen (s. o.).

Weiterhin fällt auf, dass der Standpunktfähigkeit von den Befragten eine weit größere Aufmerksamkeit gewidmet wird als dem Perspektivenwechsel. Das ergibt nicht nur die Anzahl der einschlägigen Belegstellen, die aufgrund der qualitativen Anlage der Studie allerdings nur eine bedingte Aussagekraft hat. Für diesen Befund spricht insbesondere, dass sich nahezu sämtliche Belege, die mit „Perspektivenwechsel“ kodiert wurden, auch mit „Standpunktfähigkeit" kodieren ließen, nicht jedoch umgekehrt. Es kann hier nicht im Detail abgeschätzt werden, inwiefern lokale Gründe für diese gesteigerte Aufmerksamkeit verantwortlich sind, wie etwa ein möglicher Fokus in den Fortbildungen etc. Angesichts der religionspädagogischen Debatte um die Standpunktfähigkeit im Allgemeinen, die sich an den sog. „Innenansichten des Religionsunterrichts“ (Englert, Hennecke \& Kämmerling, 2014) entzündet hat (z. B. Grönefeld, 2018; Heimbrock, 2017; Lüdtke, 2020, S. 136-150; Zimmermann, 2020), könnte das Bewusstsein um die eigene Konfessionalität oder das In-Szene-Setzen derselben im Religionsunterricht auch der Problematik geschuldet sein, die eine solche Anforderung für Religionslehrpersonen praktisch aufwirft. 
Die vorliegenden Befunde geben dieser Problematik eine Kontur. Zuerst kann sich Standpunktfähigkeit entlang dreier Ebenen ausdrücken, nämlich in den religiösen Einstellungen der Lehrperson, in ihrem Wissen über die beiden Konfessionen und in ihrem Verhalten im Religionsunterricht. Alle drei Ebenen haben die Befragten im Blick. Berücksichtigt man die Befunde zum Perspektivenwechsel, scheint es nur auf der kognitiven Ebene die Möglichkeit zu geben, auch den Standpunkt der jeweils anderen Konfession einzunehmen - und zwar im Modus der Information über konfessionsspezifische Sachverhalte. Sowohl bei den Einstellungen als auch beim Verhalten scheint dagegen die Konfession, in der man aufgewachsen ist, den Rahmen der Möglichkeiten abzustecken. In beiden Fällen fallen immer wieder die Begriffe der "Authentizität“ oder der "Zeugenschaft", was die existentielle Verwobenheit des eigenen Standpunkts auf beiden Ebenen unterstreicht. In allen diesen Aspekten stimmt die Wahrnehmung der Befragten mit den kirchlichen (DBK, 1996; EKD, 1994) und religionspädagogischen Konzepten überein (z. B. Heger, 2021; Ziebertz, 2010). Die unter modernen Bedingungen durchaus denkbare Möglichkeit, dass die Auseinandersetzung mit anderen Konfessionen auch zu einer Verschiebung des Standpunktes sowohl hinsichtlich der eigenen Überzeugung als auch der Performance im Unterricht in eine dieser Konfessionen hinein geschieht, scheint für die Befragten nicht im Blickfeld zu sein.

Sehr klar ist den Befragten jedoch, dass mit dem individuellen Glauben und der kollektiven Perspektive der eigenen Kirche zwei mögliche Bezugspunkte für den eigenen Standpunkt vorliegen. Theologisch betrachtet mag diese Differenz artifiziell sein (z. B. Schweitzer, 2017). Im modernen Mind-Set stellen sie jedoch zwei nicht notwendig miteinander vereinbare Bezugsgrößen dar, was für die Befragten insbesondere im Religionsunterricht eine Herausforderung sein kann. Dieses Dilemma ist nicht neu (z. B. Schach, 1980), scheint im kokoRU auf Grund des spezifischen konfessionellen Fokus aber eine eigene Bedeutung zu entfalten. Es wäre eine eigene Untersuchung wert, ob sich diese Problematik für römisch-katholische Lehrkräfte häufiger stellt als für evangelische. Eine solche Vermutung legen zumindest die Ekklesiologien beider Konfessionen nahe.

Theoretisch instruktiv erweisen sich ferner die beiden kritischen Einlassungen zur Standpunktfähigkeit (vgl. 3.3). Der Verweis auf die Re-Ideologisierung im zweiten O-Ton scheint angesichts des kommunizierten Zielspektrums des kokoRU auf den ersten Blick an der Sache vorbei zu gehen, denn dieses betont eindeutig die Mündigkeit der Schülerinnen und Schüler. Allerdings finden sich zumindest in den katholischen Texten zu dieser Organisationsform immer wieder Verweise auf das sog. „Eigene“ der Kinder und Jugendlichen. So schreibt die DBK (1996, S. 22): „Sie [die Schülerinnen und Schüler] werden durch die Begegnung mit der anderen Konfession angeregt, sich ihrer eigenen konfessionellen Prägung und Kirchenzugehörigkeit bewusst zu werden und diese zu reflektieren." Und Klaus Kießling, Andreas Günter und Stephan Pruchniewicz (2018, S. 130) assistieren: „Konfessionelles Selbstbewusstsein und konfessionelle Sprach- bzw. Ausdrucksfähigkeit müssen in einem ersten Schritt vor allem als Lernziele des kooperativen Religionsunterrichts gefasst werden.“ Darüber hinaus finden sich religionspädagogische Stimmen, die die ökumenische Grundhaltung des konfessionell-kooperativen Lernsettings einklagen (z. B. LinkWieczorek, 2017; Möller \& Wedding, 2017; Woppowa \& Zimmermann, 2018). Weitere Evaluationen müssen zeigen, in welche Richtung sich der kokoRU bewegt.

Schließlich verweist die Einlassung zur Heterogenität konfessioneller Orientierungen an die Komplexität individueller und kollektiver Identitäten (z. B. Boschki, 2017). So sehr die religiösen Gemeinschaften einen Lernraum für religiöse Bildung aufspannen (Gärtner, 2015, S. 90-93), so divers und vielgestaltig erweist sich dieser Raum bei näherer Betrachtung. Unseres Erachtens markiert obige Beobachtung aus einer Gruppendiskussion einen Ausweg aus dem von manchen Kolleginnen und Kollegen erfahrenen Dilemma, die eigene Religiosität mit der kirchlichen Identität unter einen Hut zu bringen. In dem Maß, in dem auch eine kirchliche Identität als vielgestaltig - nicht: beliebig - ausgewiesen wird, in dem Maß relativiert sich eine mögliche Unvereinbarkeit mit der individuellen Religiosität. Wenn Jan Woppowa (2021, S. 203) jedoch vor einer „konfessionellen Überbeanspruchung“ warnt und dieses Thema bei einer Gruppendiskussion zum kokoRU aufkommt, scheint dieser Sachverhalt noch nicht hinreichend kommuniziert zu sein.

\section{Literaturverzeichnis}

Bauer, Jochen (2019). Religionsunterricht für alle. Eine multitheologische Fachdidaktik. Stuttgart: Kohlhammer.

Boschki, Reinhold (2017). „Aldi oder Lidl?“ - Konfessionelle Differenz und Identitätskonstruktion in Zeiten konfessioneller Indifferenz. In Konstantin Lindner, Mirjam Schambeck, Henrik Simojoki \& Elisabeth Naurath (Hg.), Zukunftsfähiger Religionsunterricht. Konfessionell - kooperativ - kontextuell (S. 159-173). Freiburg i. Br.: Herder. 
Caspary, Christiane (2016). Umgang mit konfessioneller Differenz im Religionsunterricht. Eine Studie zur Didaktik des konfessionell-kooperativen Religionsunterrichts. Münster: LIT.

Die Deutschen Bischöfe [DBK] (Hg.) (1996). Die bildende Kraft des Religionsunterrichts. Zur Konfessionalität des katholischen Religionsunterrichts. Bonn: DBK.

Die Deutschen Bischöfe [DBK] (Hg.) (2016). Die Zukunft des konfessionellen Religionsunterrichts. Empfehlungen für die Kooperation des katholischen mit dem evangelischen Religionsunterricht. Bonn: DBK.

Evangelische Kirche in Deutschland [EKD] (Hg.) (1994). Identität und Verständigung. Standort und Perspektiven des Religionsunterrichts in der Pluralität. Gütersloh: Gütersloher Verlagshaus.

Englert, Rudolf; Hennecke, Elisabeth \& Kämmerling, Markus (2014). Innenansichten des Religionsunterrichts. Fallbeispiele - Analysen - Konsequenzen. München: Kösel.

Gärtner, Claudia (2015). Religionsunterricht - ein Auslaufmodell? Begründung und Grundlagen religiöser Bildung in der Schule. Paderborn: Ferdinand Schöningh.

Gennerich, Carsten \& Mokrosch, Reinhold (2016). Religionsunterricht kooperativ. Evaluation des konfessionell-kooperativen Religionsunterrichts in Niedersachsen und Perspektiven für einen religions-kooperativen Unterricht. Stuttgart: Kohlhammer.

Grönefeld, Michael (2018). RU als Kommunikationsraum einer Vielfalt an Anschauungen. Katechetische Blätter, 143(2), 85-88.

Heger, Johannes (2021). Lehrpersonen als Vertreterinnen und Vertreter der Kirche. In Ulrich Kropač \& Ulrich Riegel (Hg.), Handbuch Religionsdidaktik (S. 144-150). Stuttgart: Kohlhammer.

Heimbrock, Hans-Günter (2017) (Ed.). Taking Position: Empirical studies and theoretical reflections on Religious Education and worldview. Münster: Waxmann.

Heimbrock, Hans-Günter \& Kerntke, Felix (2017). Evangelisches Profil im Widerspruch. Gelebte Konfessionalität von Religionslehrern in der EKHN. Eine empirische Untersuchung. In Hans-Günter Heimbrock (Ed.), Taking Position: Empirical studies and theoretical reflections on Religious Education and worldview (p. 23-80). Münster: Waxmann.

Isak, Rainer (2009). KRU im Spiegel der Äußerungen von Lehrerinnen und Lehrern. In Lothar Kuld, Friedrich Schweitzer, Werner Tzscheetzsch \& Joachim Weinhardt (Hg.), Im Religionsunterricht zusammenarbeiten. Evaluation des konfessionell-kooperativen Religionsunterrichts in Baden-Württemberg (S. 134-169). Stuttgart: Kohlhammer.

Kießling, Klaus; Günter, Andreas \& Pruchniewicz, Stephan (2018). Machen Unterschiede Unterschiede? Konfessioneller Religionsunterricht in gemischten Lerngruppen. Ansichten - Einsichten - Aussichten. Göttingen: Vandenhoeck \& Ruprecht.

Kuld, Lothar; Schweitzer, Friedrich; Tzscheetzsch, Werner \& Weinhardt, Joachim (2009). Im Religionsunterricht zusammenarbeiten. Evaluation des konfessionell-kooperativen Religionsunterrichts in Baden-Württemberg. Stuttgart: Kohlhammer.

Lindner, Konstantin \& Simojoki, Henrik (2018). Konfessionelle Kooperation - didaktisch. Warum, wie und was es noch braucht. Katechetische Blätter, 143(2), 91-95.

Link-Wieczorek, Ulrike (2017). Im Religionsunterricht konfessionell kooperieren. Ökumenisch-theologische Grundlegung. In Konstantin Lindner, Mirjam Schambeck, Henrik Simojoki \& Elisabeth Naurath (Hg.), Zukunftsfähiger Religionsunterricht. Konfessionell - kooperativ - kontextuell (S. 123-138). Freiburg: Herder.

Lüdtke, Antonia (2020). Confessional Gap. Konfessionalität und Religionsunterricht denken. Stuttgart: Kohlhammer.

Mayring, Philipp (2015). Qualitative Inhaltsanalyse. Grundlagen und Techniken (12. Auflage). Weinheim: Beltz.

Möller, Rainer \& Wedding, Michael (2017). Mehr Communio- bzw. Ökumenesensibilität! - oder: Wird das Differenzkonzept religionspädagogisch überstrapaziert? In Konstantin Lindner, Mirjam Schambeck, Henrik Simojoki \& Elisabeth Naurath (Hg.), Zukunftsfähiger Religionsunterricht. Konfessionell - kooperativ - kontextuell (S. 139-158). Freiburg i. Br.: Herder.

Platzbecker, Paul (2018). Der konfessionell-kooperative Religionsunterricht in NRW. ,Kokolores' oder ,Modell der Zukunft'? Religionspädagogische Beiträge, 78, 33-44.

Pohl-Patalong, Uta; Woyke, Johannes; Boll, Stefanie; Dittrich, Thorsten \& Lüdtke, Antonia E. (2016). Konfessioneller Religionsunterricht in religiöser Vielfalt. Eine empirische Studie zum evangelischen Religionsunterricht in SchleswigHolstein. Stuttgart: Kohlhammer.

Rothgangel, Martin; Lück, Christhard \& Klutz, Philipp (2017). Praxis Religionsunterricht. Einstellungen, Wahrnehmungen und Präferenzen von ReligionslehrerInnen. Stuttgart: Kohlhammer.

Sajak, Clauß Peter (2017). Art. Konfessionell-kooperativer Religionsunterricht. Das wissenschaftliche Lexikon im Inter- 
net. https://doi.org/10.23768/wirelex.Konfessionellkooperativer Religionsunterricht.100235

Schach, Bernhard (1980). Der Religionslehrer im Rollenkonflikt: Eine religionssoziologische Untersuchung. München: Kösel.

Schambeck, Mirjam \& Schröder, Bernd (2017). Auf dem Weg zu einer Didaktik konfessionell-kooperativer Lernprozesse. In Konstantin Lindner, Mirjam Schambeck, Henrik Simojoki \& Elisabeth Naurath (Hg.), Zukunftsfähiger Religionsunterricht. Konfessionell - kooperativ - kontextuell (S. 343-363). Freiburg i. Br.: Herder.

Schmid, Hans (2017). Die Bedeutung des Konfessorischen in einem zukunftsfähigen Religionsunterricht aus katholischer Sicht. In Konstantin Lindner, Mirjam Schambeck, Henrik Simojoki \& Elisabeth Naurath (Hg.), Zukunftsfähiger Religionsunterricht. Konfessionell - kooperativ - kontextuell (S. 55-66). Freiburg i. Br.: Herder.

Schröder, Bernd \& Biesinger, Albert (2016). Konfessionelle Kooperation und der Stand ihrer religionspädagogischen Erforschung. In Stefan Altmeyer, Rudolf Englert, Helga Kohler-Spiegel, Elisabeth Naurath, Bernd Schröder \& Friedrich Schweitzer (Hg.), Ökumene im Religionsunterricht (S. 73-86). Göttingen: Vandenhoeck \& Ruprecht.

Schweitzer, Friedrich (2017). Die Bedeutung von Konfessionalität für einen zukunftsfähigen Religionsunterricht aus evangelischer Sicht. In Konstantin Lindner, Mirjam Schambeck, Henrik Simojoki \& Elisabeth Naurath (Hg.), Zukunftsfähiger Religionsunterricht. Konfessionell - kooperativ - kontextuell (S. 41-54). Freiburg i. Br.: Herder.

Schweitzer, Friedrich \& Biesinger, Albert (2002). Gemeinsamkeiten stärken - Unterschieden gerecht werden. Erfahrungen und Perspektiven zum konfessionell-kooperativen Religionsunterricht. Freiburg i. Br.: Herder/Gütersloh: Gütersloher Verlagshaus.

Schweitzer, Friedrich; Biesinger, Albert; Conrad, Jörg \& Gronover, Matthias (2006). Dialogischer Religionsunterricht. Analyse und Praxis konfessionell-kooperativen Religionsunterrichts im Jugendalter. Freiburg i. Br.: Herder.

Woppowa, Jan (2015). Grundlegung einer Didaktik der konfessionellen Kooperation im schulischen Religionsunterricht. In Jan Woppowa (Hg.), Perspektiven wechseln. Lernsequenzen für den konfessionell-kooperativen Religionsunterricht (S. 5-17). Paderborn: Ferdinand Schöningh.

Woppowa, Jan (2017). Perspektivenverschränkung als zentrale Figur konfessioneller Kooperation. In Konstantin Lindner, Mirjam Schambeck, Henrik Simojoki \& Elisabeth Naurath (Hg.), Zukunftsfähiger Religionsunterricht. Konfessionell - kooperativ - kontextuell (S. 174-192). Freiburg i. Br.: Herder.

Woppowa, Jan (2021). Konfessionell-kooperativer Religionsunterricht. In Ulrich Kropač \& Ulrich Riegel (Hg.), Handbuch Religionsdidaktik (S. 198-204). Stuttgart: Kohlhammer.

Woppowa, Jan \& Zimmermann, Mirjam (2018). Konfessionelle Identitätsmarker?! Die Chancen konfessionell-kooperativen Unterrichts. Religion 5-10, 32(4), 4-7.

Ziebertz, Hans-Georg (2010). Wer initiiert religiöse Lernprozesse? - Rolle und Person der Religionslehrerinnen und -lehrer. In Georg Hilger, Stephan Leimgruber \& Hans-Georg Ziebertz (Hg.), Religionsdidaktik (S. 206-226). München: Kösel.

Zimmermann, Mirjam (2020). In der (konfessionellen) Selbstauflösung? Zum Berufsverständnis von Religionslehrerinnen und Religionslehrern in religionspädagogischen Handlungsfeldern - Betrachtungen aus der Innenund Außenperspektive. In Bernd Schröder \& Thomas Schlag (Hg.), Praktische Theologie und Religionspädagogik (S. 377-412). Leipzig: Evangelische Verlagsanstalt. 